\title{
Age-Related Thinning of Bowman's Layer in the Human Cornea In Vivo
}

\author{
Johan Germundsson, Georgios Karanis, Per Fagerholm and Neil Lagali
}

\section{Linköping University Post Print}

\section{Tweet}

N.B.: When citing this work, cite the original article.

Original Publication:

Johan Germundsson, Georgios Karanis, Per Fagerholm and Neil Lagali, Age-Related Thinning of Bowman's Layer in the Human Cornea In Vivo, 2013, Investigative Ophthalmology and Visual Science, (54), 9, 6143-6149.

http://dx.doi.org/10.1167/iovs.13-12535

Copyright: Association for Research in Vision and Ophthalmology (ARVO) http://www.arvo.org/ 
Age-related thinning of Bowman's layer in the human cornea in vivo

Johan Germundsson, Georgios Karanis, Per Fagerholm, and Neil Lagali

Department of Clinical and Experimental Medicine - Ophthalmology

Faculty of Health Sciences, Linköping University, 58183 Linköping, Sweden

Abstract word count: 242

Word count: 3090

Sources of Funding: Funding from the Cronqvist Foundation to NL, and funding from the Swedish Research Council, Konung Gustav V and Drottning Viktorias Frimurarestiftelse, and The County Council of Östergötland to PF.

None of the authors have any proprietary/financial interest to disclose.

Running head: Age related thinning of Bowman's layer

Corresponding author:

Neil Lagali, $\mathrm{PhD}$

Department of Clinical and Experimental Medicine - Ophthalmology

Faculty of Health Sciences

Linköping University

58183 Linköping, Sweden

neil.lagali@liu.se

Tel +46101034658

Fax +46101033065 


\section{ABSTRACT}

Purpose: To determine the thickness of Bowman's layer (BL) in vivo in a normal, healthy population, and to determine its variation with age.

Methods: 82 subjects aged 15 - 88y with clear, healthy corneas were examined bilaterally with laser-scanning in vivo confocal microscopy (IVCM). BL thickness was determined from IVCM images of anterior and posterior BL boundaries. For a given eye, BL thickness was averaged across four central locations by two independent observers. Additionally, central corneal thickness was measured by time-domain optical coherence tomography (OCT).

Results: A significant negative correlation of BL thickness with age was found in both right (Pearson $r=-0.579, \mathrm{P}<0.0001)$ and left $(\mathrm{r}=-0.558, \mathrm{P}<0.0001)$ eyes. Linear regression analysis yielded a decline in BL thickness of $0.06 \mu \mathrm{m} /$ year. Older subjects (mean $64.4 \mathrm{y} ; \mathrm{N}=$ 41) had a mean BL thickness ( $8.6 \pm 1.7 \mu \mathrm{m}$, RE) significantly thinner than younger subjects (mean 31.6y; $\mathrm{N}=41 ; 10.7 \pm 1.6 \mu \mathrm{m}$, RE; $\mathrm{P}<0.001$ ). No correlation of corneal thickness with age or BL thickness with corneal thickness was found. Strong inter-eye correlations in BL thickness (Pearson $r=0.771, P<0.0001)$ and corneal thickness $(r=0.969, P<0.001)$ were found.

Conclusions: BL thins with age in the normal cornea, losing one-third of its thickness between the ages of 20 and 80. In vivo measurement of BL thickness by IVCM could aid in clinical assessment and planning treatments of the anterior cornea. 


\section{Introduction}

Bowman's layer (BL) is a normally acellular layer located immediately posterior to the corneal epithelium. BL is structurally composed of collagen fibrils, smaller in diameter and randomly oriented, compared to stromal collagen where fibrils are larger and directionally oriented in parallel lamellae. ${ }^{1,2}$ Bowman's layer's functional role is not completely known, but it is believed to serve as a barrier that protects the corneal stroma from traumatic injury. ${ }^{3-5}$ In this manner, BL aids in the maintenance of corneal transparency and epithelial innervation in cases of injury, disease, or surgical intervention. ${ }^{5}$

The protective nature of Bowman's layer may be related to its structural strength and its thickness. Knowledge of the BL thickness could not only enable an assessment of its protective ability, but this parameter is also of importance when planning various types of laser surgery. ${ }^{5-7}$ Much discrepancy, however, exists in published values of mean BL thickness in humans, with values ranging from 8 to $19 \mu \mathrm{m}$, depending on the type of measurement (in vivo or ex vivo), tissue preparation method, and the measurement technique. ${ }^{2,7-13}$

Recently, we developed an accurate method to measure BL in vivo, based on laser-scanning in vivo confocal microscopy (IVCM) ${ }^{14}$ By this method, three-dimensional laser-scanning confocal depth scans of BL are taken in live subjects, and an image analysis procedure is used to determine the upper and lower boundaries of BL. In contrast to other in vivo techniques where reflectivity of epithelial and stromal interfaces is used to infer BL thickness, our method directly examines morphologic features of BL to determine its boundaries.

To date there are no studies examining BL thickness in a large healthy population, due to the difficulty in obtaining healthy donor cornea tissue from a wide age range, unknown effects of tissue fixation and shrinkage of BL, and the difficulty in accurately measuring this thin layer in vivo with lower resolution techniques. In this study, we used laser-scanning IVCM to accurately measure BL thickness in the central cornea in a large, healthy population with a wide age range.

\section{Methods}

\section{Recruitment and clinical examination of subjects}

After obtaining ethical approval from the regional ethical review board in Linköping, and with signed informed consent, 115 healthy volunteers were recruited for this study. The study adhered to the tenets of the Declaration of Helsinki. Volunteers were recruited from the population of persons accompanying patients to the Department of Ophthalmology at Linköping University. Each volunteer underwent full ophthalmic examination in both eyes including slit lamp biomicroscopy, intraocular pressure, refraction, best spectacle-corrected visual acuity (BSCVA), in vivo confocal microscopy and anterior segment optical coherence tomography (OCT). Individuals with ocular pathology or with diabetes, a history of contact 
lens wear, prior corneal surgery, or taking eye medications were excluded. No further selection criteria was initially used; however, as the study progressed individuals were selected based on age to achieve a target final cohort consisting of 4 age groups of 20 individuals each (15-30y, 31-45y, 46-60y, 61+y).

\section{Measurement of central corneal thickness}

Using anterior segment optical coherence tomography (Visante ${ }^{\circledR}$, Carl Zeiss Meditec, Jena, Germany) all participants underwent a global high resolution pachymetry scan bilaterally prior to IVCM examination. Central corneal thickness was taken as the mean value in the central $2 \mathrm{~mm}$ diameter zone as determined by the OCT software.

\section{In vivo confocal microscopy of Bowman's layer}

An in vivo confocal microscope (HRT3-RCM, Heidelberg Engineering, Heidelberg, Germany) was used to conduct all examinations; details of the IVCM procedure have been described elsewhere. ${ }^{14,15}$ Subjects were examined bilaterally and for each examined eye, up to 10 confocal scans were obtained. Scans were taken in both volume and section scan modes. In the volume scan mode, depth is automatically scanned by an internal motor, to obtain $\mathbf{4 0}$ images spanning a thickness of $80 \mu \mathrm{m}$. The volume scan mode was initiated when a central corneal wing cell layer was visible in the real-time image display, and the scan automatically terminated in the anterior stroma. The section scan mode was used to acquire scans with 100 images at 8 frames/sec, while the focal depth was varied manually to cross Bowman's layer 2-3 times during the scan. Manual depth adjustment was facilitated by the use of a joystickdriven motor control. After each volume or section scan, the lateral positioning of the microscope objective was adjusted (in a random direction, but keeping within the nonoblique central zone) to image a different central location, and scanning was repeated. Typically several central corneal locations were scanned for each eye, in order to sample a larger region of the central cornea. Because the volume scans resulted in more closely spaced images axially (separated by approximately $2 \mu \mathrm{m}$ in depth), the majority of scans taken were volume scans.

\section{Measurement of Bowman's layer thickness}

IVCM scans were used to determine BL thickness based on a previously reported method, ${ }^{14}$ but with the following adjustments. First, images from volume scans were used where possible, and image sequences with obvious motion artifacts (lateral and/or axial) were excluded. Also, obviously tilted (oblique) images and images with extensive artifacts due to mechanical pressure on the cornea were excluded. Two observers selected the final scans for further analysis. Scans were ordered in a randomized manner, with observers masked to the patient age. Next, the selected scans were exported as sets of individual images without image depth or patient identifying information. For each sequence of images, only the morphologic features in a small sub-region of the image frame were considered, to further avoid the influence of image obliqueness and pressure-induced artifacts. The same sub-region of the image was observed in successive image frames to identify the features defining the anterior and posterior limits of Bowman's layer. The anterior limit of BL was considered as 
the most anterior epithelial layer with haze (often with a slight haze and subbasal nerves visible). The posterior limit of BL was considered as the most anterior layer of stroma with haze where indistinct keratocytes were visible. The image numbers corresponding to the anterior and posterior BL limits were recorded, and only later was the image depth unmasked. BL thickness was determined independently by two trained observers. For each eye, a mean BL thickness was calculated from measurements of thickness in approximately 4 different regions in the central cornea.

\section{Quantitative analysis and statistics}

The Bland-Altman method ${ }^{16}$ was used to compare differences in BL thickness measurements between the two independent observers. Inter-observer differences are reported as the $95 \%$ limits of agreement (LOA).

Inter-observer correlation of BL thickness and correlation of central corneal thickness or BL thickness with eye and age were determined by the Pearson correlation test. Difference in BL thickness across age groups was tested with one-way ANOVA and the t-test. Differences in corneal thickness or BL thickness in male and female subjects were tested with the t-test. Statistical and regression analyses were performed using the software package SigmaStat 3.5 for Windows, and two-tailed P-values of less than 0.05 were considered significant.

\section{Results}

\section{Subject characteristics}

Of the 115 examined volunteers, 82 subjects (164 eyes) were included in the study. Patients were excluded due to the presence of corneal haze, localized corneal scarring, endothelial pigment deposits, diabetes, corneal dystrophy, dry eye, prior corneal trauma or surgery, or IVCM images without sufficient quality for analysis. Characteristics for the subjects included in the study are summarized in Table 1 . Mean subject age was 48 years at the time of examination (range 15 - 88y). Roughly equal numbers of participants were examined in each age category.

Of the 164 eyes examined, 67 right eyes were emmetropic (spherical equivalent refraction $>$ $0.5 \mathrm{D}$ but $<+1.5 \mathrm{D}), 8$ were hyperopic $(\geq+1.5 \mathrm{D})$ and 7 were myopic $(\leq-0.5 \mathrm{D})$. 68 left eyes were emmetropic, 7 were hyperopic and 7 were myopic. 53 of the 82 subjects had a bilateral uncorrected visual acuity of 1.0 (20/20 Snellen equivalent). Mean and standard deviation of decimal BSCVA was $0.99 \pm 0.3$ lines (right eyes) and $0.98 \pm 0.3$ lines (left eyes). Mean and standard deviation of intraocular pressure was $15.4 \pm 2.8 \mathrm{mmHg}$ (right eyes) and $15.5 \pm 3.0$ mmHg (left eyes). 
Table 1. Demographic characteristics of healthy volunteer subjects.

\begin{tabular}{|c|c|c|c|c|}
\hline & \multicolumn{4}{|c|}{ Total group } \\
\hline No. of subjects & \multicolumn{4}{|c|}{82} \\
\hline Female & \multicolumn{4}{|c|}{45} \\
\hline Male & \multicolumn{4}{|c|}{37} \\
\hline Mean age (y) & \multicolumn{4}{|c|}{48.0} \\
\hline Female & \multicolumn{4}{|c|}{48.9} \\
\hline male & \multicolumn{4}{|c|}{46.9} \\
\hline Age group & $15-30$ & $31-45$ & $46-60$ & $61+$ \\
\hline No. of subjects & 20 & 20 & 18 & 24 \\
\hline mean age & 25.1 & 37.4 & 54.4 & 71.2 \\
\hline female & 10 & 8 & 15 & 12 \\
\hline male & 10 & 12 & 3 & 12 \\
\hline
\end{tabular}

\section{Central corneal thickness}

Central corneal thickness by OCT was $535.4 \pm 31.3 \mu \mathrm{m}$ (RE, mean \pm SD) and $533.9 \pm 31.2 \mu \mathrm{m}$ (LE). Thickness in right and left eyes was highly correlated (Pearson $\mathrm{r}=0.969, \mathrm{P}<0.001$ ). No significant correlation of central corneal thickness was found with age in either right $(\mathrm{r}=\mathrm{-}$ 0.038, $\mathrm{P}=0.74)$ or left $(\mathrm{r}=0.020, \mathrm{P}=0.86)$ eyes (Fig 1$)$; however, the standard deviation increased with increasing age (Table 2). No difference in corneal thickness between female and male subjects was found for either eye.

Table 2. Central corneal thickness by OCT, grouped by age and eye.

\begin{tabular}{cc|c|c|c} 
& \multicolumn{4}{c}{ Age group } \\
\cline { 2 - 5 } Eye & $15-30 y$ & $31-45 y$ & $46-60 y$ & $61 y+$ \\
\hline RE & $534 \pm 27$ & $542 \pm 30$ & $537 \pm 34$ & $532 \pm 35$ \\
LE & $531 \pm 28$ & $539 \pm 27$ & $538 \pm 33$ & $530 \pm 36$
\end{tabular}

\section{Inter-observer differences in Bowman's layer thickness}

For each subject and eye, IVCM image scans were analyzed to select those which did not contain significant motion-induced artifacts (in axial or lateral directions). For all eyes, 2 to 5 image scans contained suitably stable images for determination of BL thickness at different central corneal locations. Data from volume scans were used to determine BL thickness; however, in some cases where suitable volume scans were not available, section scans were used. In the 82 subjects, BL thickness was determined at a mean of 3.9 different central corneal locations in the right eye and at a mean of 3.7 in the left eye. For each eye, a single mean central BL thickness was determined by averaging values from the different locations. The mean value for each eye was compared between observers (Fig. 2). The values between observers were highly correlated $(\mathrm{P}<0.001$ for RE and LE), however, inclusion of the slope 
$=1$ line in Fig. 2 indicated a tendency of Observer 1 to record slightly thicker values than Observer 2 in cases where BL thickness exceeded $10 \mu \mathrm{m}$.

Bland-Altman analysis indicated a mean difference of $0.05 \mu \mathrm{m}$ in BL thickness between observers in the RE and $0.11 \mu \mathrm{m}$ in the LE. The 95\% LOA were $\pm 3.4 \mu \mathrm{m}$ and $\pm 3.0 \mu \mathrm{m}$ for RE and LE, respectively (Fig. 3).

\section{Age-dependence of BL thickness}

Values of BL thickness for each eye were averaged across observers to yield a single value for central BL thickness. The resulting BL thickness was significantly negatively correlated with subject age in right $(r=-0.579, \mathrm{P}<0.0001)$ and left $(\mathrm{r}=-0.558, \mathrm{P}<0.0001)$ eyes (Fig. 4). Additionally, BL thickness in right and left eyes of the same subject was highly correlated ( $r=0.771, P<0.0001)$. Regression analysis yielded the following formulae for BL thickness:

BL thickness $(\mu \mathrm{m})=12.50-0.060 \times$ age $(y)$

BL thickness $(\mu \mathrm{m})=12.66-0.065 \times$ age $(y)$

which corresponds to a decline in thickness of about $0.06 \mu \mathrm{m} /$ year.

When compiled according to the four age categories, a clear decline in mean BL thickness was evident (Table 3). As age increased, the minimum and maximum BL thickness in the age group generally decreased, while the variation of BL thickness within a group remained at a constant 6 -7 $\mu \mathrm{m}$. One-way ANOVA across age categories indicated a significant age dependence of BL thickness ( $\mathrm{P}<0.001$ for both $\mathrm{RE}$ and $\mathrm{LE}$ ); however, pairwise comparisons indicated no significant difference between the two youngest or between the two eldest age categories, independent of eye $(\mathrm{P}>0.05)$. Age categories were then collapsed into two categories consisting of subjects younger than or older than (or equal to) the mean age of 48y. The number of subjects in each of the two categories was equal (41 subjects). For right and left eyes, t-tests indicated a significantly thinner BL for older subjects $(64.4 \pm 9.3 \mathrm{y}$; RE: $8.6 \pm$ $1.7 \mu \mathrm{m}$, mean $\pm \mathrm{SD}$; LE: $8.4 \pm 1.9 \mu \mathrm{m})$ relative to younger subjects $(31.6 \pm 7.6 \mathrm{y} ; \mathrm{RE}: 10.7 \pm$ $1.6 \mu \mathrm{m}$; LE: $10.7 \pm 1.8 \mu \mathrm{m}) ; \mathrm{P}<0.001$ for RE and $\mathrm{P}<0.001$ for LE. No difference in $\mathrm{BL}$ thickness was found between male and female subjects for either eye.

\begin{tabular}{c|c|c|c|c|c} 
& \multicolumn{5}{|c}{ Age group } \\
& Eye & $15-30 y$ & $31-45 y$ & $46-60 y$ & $61+y$ \\
\cline { 2 - 6 } Mean BL thickness $(\mu \mathrm{m})$ & RE & $11.0 \pm 1.7$ & $10.5 \pm 1.4$ & $9.1 \pm 1.8$ & $8.3 \pm 1.6$ \\
& LE & $11.0 \pm 2.2$ & $10.5 \pm 1.3$ & $8.9 \pm 2.0$ & $8.0 \pm 1.7$ \\
range $(\mu \mathrm{m})$ & both & $7.5-14.5$ & $7.8-13.1$ & $6.0-13.2$ & $5.5-12.0$
\end{tabular}

Table 3. Central BL thickness categorized by age group. Values are given as mean \pm standard deviation. 


\section{Correlation of total corneal thickness and BL thickness}

No correlation was evident between central corneal thickness measured by OCT and central BL thickness measured by IVCM in either the RE (Pearson $r=0.10 ; \mathrm{P}=0.38$ ) or the $\mathrm{LE}(\mathrm{r}=$ $0.12 ; \mathrm{P}=0.27)$.

\section{Discussion}

In this study, the largest to date to measure in vivo BL thickness, a strong negative correlation of BL thickness with age was found. Bowman's layer becomes thinner with age, with a loss of about 32\% in thickness between the ages of 20 and 80 . Stated in another way, BL is on average $20 \%$ thinner in older subjects compared to younger subjects. Though the negative correlation of BL thickness with age was highly significant, a large variation in BL thickness across individuals was noted, confirming our previous in vivo and light microscopy measurements in smaller cohorts. ${ }^{14}$ Notably, the thinnest values for BL were approximately $50 \%$ thinner than the thickest values in the same age group. This large variation has been reported in our previous study ${ }^{14}$ and is confirmed by other studies using slit-scanning confocal microscopy ${ }^{13}$ and OCT. ${ }^{11}$ The variation of BL thickness in a normal population is much larger in relative terms, than the variation observed in total corneal thickness or epithelial thickness. ${ }^{7,8,13}$ This large variation in BL thickness may be one reason why its age dependence has not been previously reported; a correspondingly large population of normal corneas, evenly distributed in age, is required to detect correlation when variability is large.

Although the reason for this age-dependent thinning is not known, one hypothesis may be that BL collagen, as with stromal collagen, becomes naturally cross-linked with age. Because $\mathrm{BL}$ is a non-regenerating layer of the cornea, ${ }^{3}$ its collagen is not renewed (or spatially organized) by keratocytes, since BL is acellular. The gradual cross-linking of BL collagen could therefore result in gradual thinning of BL without a compensatory mechanism of new collagen production and deposition. An alternative hypothesis could be a gradual compacting of BL collagen with age due to collagen degradation, hydrostatic pressure, or some other mechanism. These hypotheses could be investigated by immunochemical methods and/or electron microscopy using a large sample of human donor corneas with wide age variation. It must be kept in mind however, that tissue preservation and fixation could lead to preparationdependent artifacts that could influence the apparent thickness.

In contrast to BL thickness, total corneal thickness was found to remain constant with age at the resolution available with the time domain OCT used. The dependence of central corneal thickness with age in normal, healthy corneas is well-investigated in studies examining large groups of individuals. ${ }^{17-20}$ A slight age dependence has been reported in some studies, while no age correlation was found in others. ${ }^{17-20}$ The values and standard deviation for central corneal thickness obtained in our Swedish population, however, agree well with the literature-reported values using ultrasound pachymetry, ${ }^{17,18,20}$ Scheimpflug photography, ${ }^{19}$ slit-scanning confocal microscopy, ${ }^{13}$ and spectral domain OCT. ${ }^{11}$ 
Although no correlation was found between BL and total corneal thickness in this study, the axial resolution of the time domain OCT used $(18 \mu \mathrm{m})^{21}$ is poorer than laser scanning IVCM $(4 \mu \mathrm{m})^{22}$. Although spectral domain OCT has a better resolution than time domain OCT, corneal thickness still cannot be measured with present instrumentation at the level of precision required $(1-2 \mu \mathrm{m})$ to detect a possible correlation with BL thickness.

Further studies are required to corroborate our BL thickness values, which are markedly thinner than other in vivo values reported. To date, in vivo BL thickness has been measured by slit-scanning confocal microscopy ${ }^{10,13}$ and spectral domain OCT. ${ }^{7,11,12}$ In these studies, however, measurements were based on interface reflectivity of the posterior epithelium and anterior stroma. It has been noted, however, that these reflective interfaces likely arise from subbasal epithelial nerves and anterior stromal keratocytes, respectively, and not the anterior and posterior aspect of BL. ${ }^{10,14}$ From the present analysis of successive image frames spaced 1-2 $\mu \mathrm{m}$ axially, it can be confirmed that each plane of maximum reflectivity is separated from a BL boundary by at least $2 \mu \mathrm{m}$, resulting in a $4 \mu \mathrm{m}$ minimum overestimation of BL thickness by reflectivity analysis.

One method to corroborate and potentially improve upon the results reported here would be to use laser-scanning IVCM in a manual scan mode with motorized joystick-driven depth adjustment. By adjusting frame acquisition rate to 30 frames/sec and the motorized depth adjustment to the slowest speed, manually scanning through a limited depth of the anterior cornea can yield images spaced $0.5 \mu \mathrm{m}$ axially. ${ }^{23}$ This approach, however, is technically challenging.

One limitation of the method used in this study is in the subjective interpretation of images for determining BL boundaries. The method was based upon earlier investigation of light and electron microscopic features of BL in the same corneas examined by IVCM; ${ }^{14}$ however, the interpretation of these borders is clearly observer-dependent, as indicated by the BlandAltman plots. In an attempt to overcome this limitation and improve the objectivity of the analysis, images were randomly and independently examined, image depth information was not available to observers until after boundaries were identified, and final thickness values were averaged across several scans and across both observers.

Another limitation was that only the central cornea was imaged. A mean of four different central corneal locations was used; however, no information on BL thickness outside the central region was available. In two studies using spectral domain OCT, spatially-resolved BL thickness measurements were reported, and local variations in BL thickness were noted. ${ }^{7,11}$ Future studies using IVCM could image mid-peripheral, peripheral and limbal locations in various sectors of the cornea to yield spatially-dependent information. The IVCM scanning procedure, however, involves considerably longer examination times compared to fast OCT scans.

Nevertheless, the strong dependence of BL thickness with age and the inter-subject variation found in the present study suggests that measurement of a patient's BL by an in vivo method could be useful in clinical assessment of the cornea. For example, treatment of epithelial 
basement membrane dystrophy by excimer laser ablation involves the removal of BL to promote epithelial anchoring. ${ }^{5}$ In this context, it is advantageous to know the exact thickness of BL to avoid unnecessary ablation of deeper stromal tissue, which could lead to scarring and nerve damage. ${ }^{5}$ Treatment of keratoconus by UVA-riboflavin collagen cross-linking may also benefit from knowledge of BL thickness, as individual variations in this layer could impact the penetration of riboflavin and/or absorption of ultraviolet energy, leading to a variation in cross-linking effect. Additionally, as BL has been thought to serve as a barrier against corneal infection ${ }^{3}$, information about its thickness could be of potential value in assessing risk or progression of infection.

\section{References}

1. Jacobsen I, Jensen O, Prause JU. Structure and composition of Bowman's membrane. Study by frozen resin cracking. Acta Ophthalmol 1984;62:39-53.

2. Komai Y, Ushiki T. The three-dimensional organization of collagen fibrils in the human cornea and sclera. Invest Ophthalmol Vis Sci 1991;32:2244-2258.

3. Wilson SE, Hong J-W. Bowman's layer structure and function. Critical or dispensable to corneal function? A hypothesis. Cornea 2000;19:417-420.

4. Obata H, Tsuru T. Corneal wound healing from the perspective of keratoplasty specimens with special reference to the function of the Bowman layer and Descemet membrane. Cornea 2007;26:S82-89.

5. Lagali N, Germundsson J, Fagerholm P. The role of Bowman's layer in corneal regeneration after phototherapeutic keratectomy: a prospective study using in vivo confocal microscopy. Invest Ophthalmol Vis Sci 2009;50:4192-8.

6. Germundssson J, Fagerholm P, Lagali N. Clinical outcome and recurrence of epithelial basement dystrophy after pthottherapeutic keratectomy. A cross-sectional study. Ophthalmology 2011;118:515-22.

7. Schmoll T, Unterhuber A, Kolbitsch C, Le T, Stingl A, Leitgeb R. Precise thickness measurements of Bowman's layer, epithelium, and tear film. Optom Vis Sci 2012;89:E795-E802.

8. Ehlers N, Heegaard S, Hjortdal J, Ivarsen A, Nielsen K, Prause JU. Morphological evaluation of normal human corneal epithelium. Acta Ophthalmol 2010;88:858-861.

9. Hayashi S, Osawa T, Tohyama K. Comparative observations on corneas, with special reference to Bowman's layer and Descemet's membrane in mammals and amphibians. $J$ Morphol 2002;254:247-258. 
10. Li HF, Petroll M, Møller-Pedersen T, Maurer JK, Cavanagh HD, Jester JV. Epithelial and corneal thickness measurements by in vivo confocal microscopy through focusing (CMTF). Curr Eye Res 1997;16:214-221.

11. Tao A, Wang J, Chen Q, Shen M, Lu F, Dubovy SR, Shousha MA. Topographic Thickness of Bowman's Layer Determined by Ultra-High Resolution Spectral DomainOptical Coherence Tomography. Invest Ophthalmol Vis Sci 2011;52:3901-7.

12. Hutchings N, Simpson TL, Hyun C, Moayed AA, Hariri S, Sorbara L, Bizheva K. Swelling of the human cornea revealed by high-speed, ultrahigh-resolution optical coherence tomography. Invest Ophthalmol Vis Sci 2010;51:4579-4584.

13. Chan KY, Cheung SW, Lam AKC, Cho P. Corneal sublayer thickness measurements with the Nidek ConfoScan 4 (Z Ring). Optom Vis Sci 2011;88:E1240-E1244.

14. Germundsson J, Fagerholm P, Koulikovska M, Lagali N. An accurate method to determine Bowman's layer thickness in vivo in the human cornea. Invest Ophthalmol Vis Sci 2012;53:2354-2359.

15. Eckard A, Stave J, Guthoff RF. In vivo investigations of the corneal epithelium with the confocal Rostock laser scanning microscope (RLSM). Cornea 2006;25:127-131.

16. Bland JM, Altman DG. Statistical methods for assessing agreement between two methods of clinical measurement. Lancet 1986;1:307-310.

17. Channa R, Mir F, Shah MN, Ali A, Ahmad K. Central corneal thickness of Pakistani adults. J Pak Med Assoc 2009;59:225-8.

18. Noche CD, Eballe AO, Bella AL. Central corneal thickness in black Cameroonian ocular hypertensive and glaucomatous subjects. Clin Ophthalmol 2010;4:1371-7.

19. Sel S, Trau S, Knak M, Kalinski T, Kaiser D, Kruse FE, Huchzermeyer C, Duncker GI, Paulsen F, Auffarth GU, Nass N. Evaluation of central corneal thickness after cataract surgery, penetrating keratoplasty and long-term soft contact lens wear. Cont Lens Anterior Eye 2013 Apr 18.

20. Gros-Otero J, Arruabarrena-Sánchez C, Teus M. Central corneal thickness in a healthy Spanish population. Arch Soc Esp Oftalmol 2011;86:73-6.

21. Maram J, Sorbara L, Simpson T. Accuracy of Visante and Zeiss-Humphrey Optical Coherence Tomographers and their cross calibration with optical pachymetry and physical references. J Optom 2011;4:147-55.

22. Niederer RL, McGhee CN. Clinical in vivo confocal microscopy of the human cornea in health and disease. Prog Retin Eye Res 2010;29:30-58. 
23. Peebo BB, Fagerholm P, Lagali N. An in vivo method for visualizing flow dynamics of cells within corneal lymphatics. Lymphat Res Biol 2013;11:93-100. 
Figures

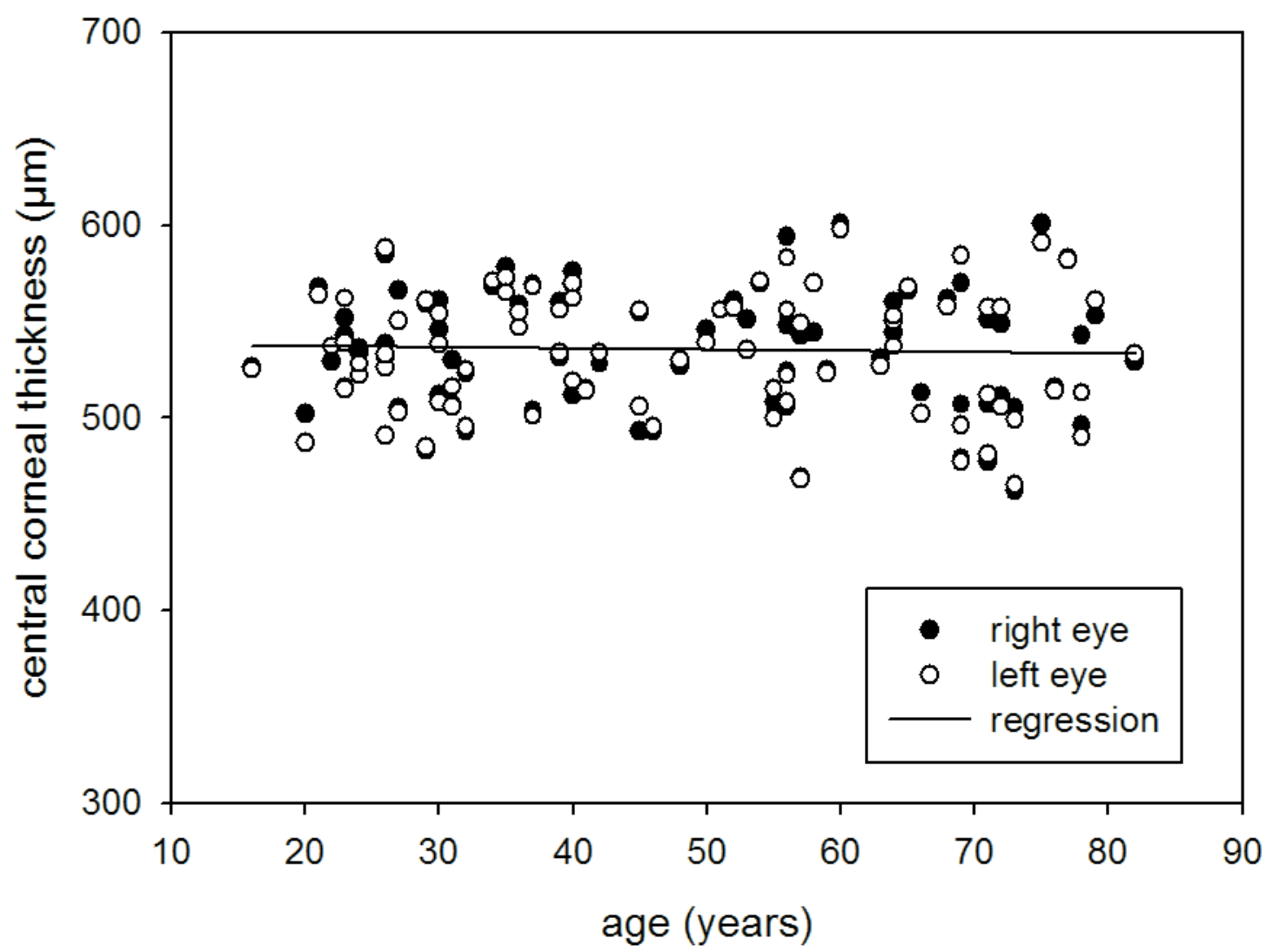

Figure 1. Variation of central corneal thickness with age, determined by OCT. The linear regression line is indicated. No correlation with age could be found in either eye, however thickness in right and left corneas in a subject were highly correlated. Standard deviation of corneal thickness increased with age. 


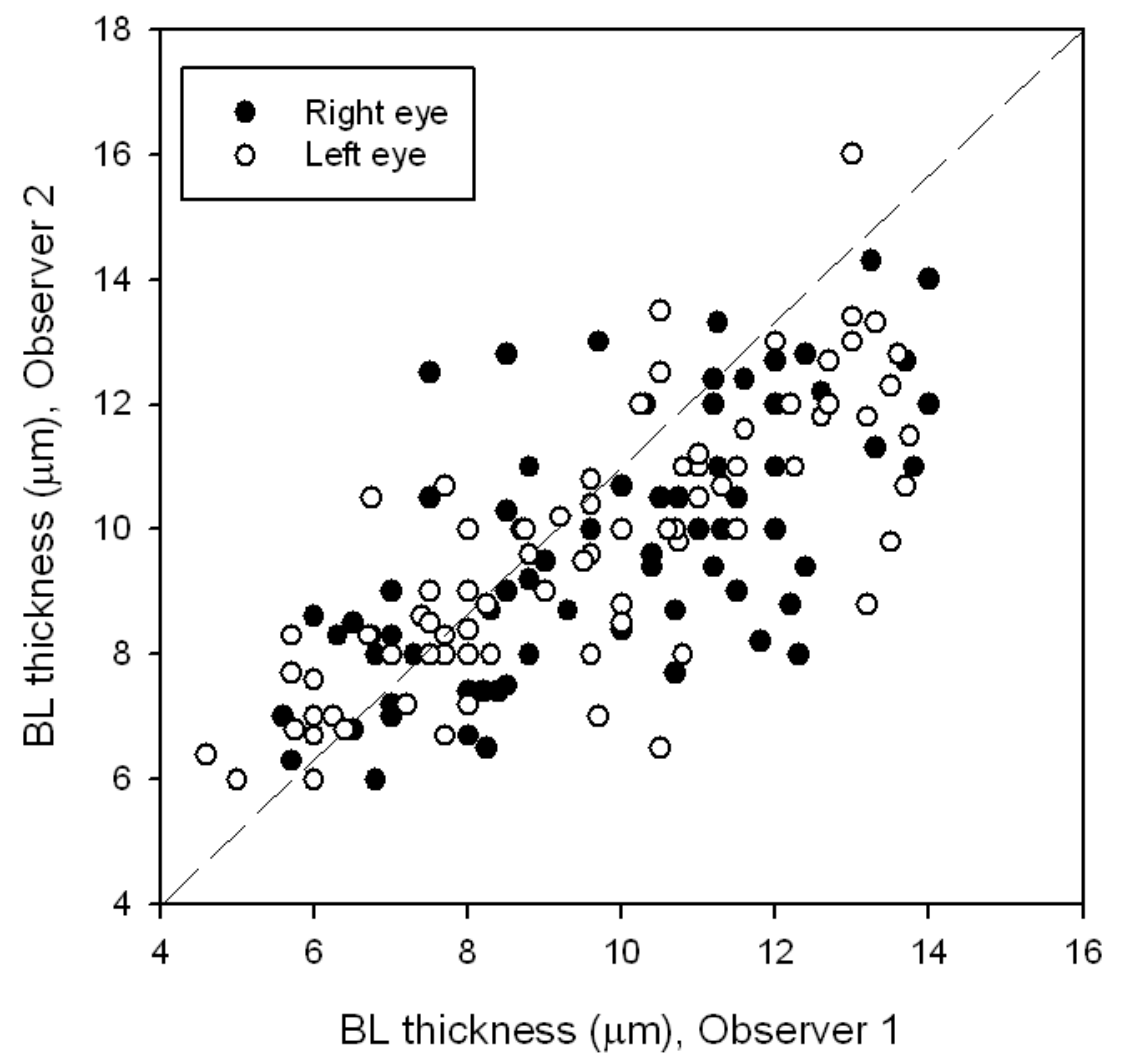

Figure 2. Correlation of BL thickness measurements between two observers. Thickness values between observers were highly correlated $(\mathrm{P}<0.001)$. The dashed line indicates the slope $=1$ line of perfect positive correlation . 

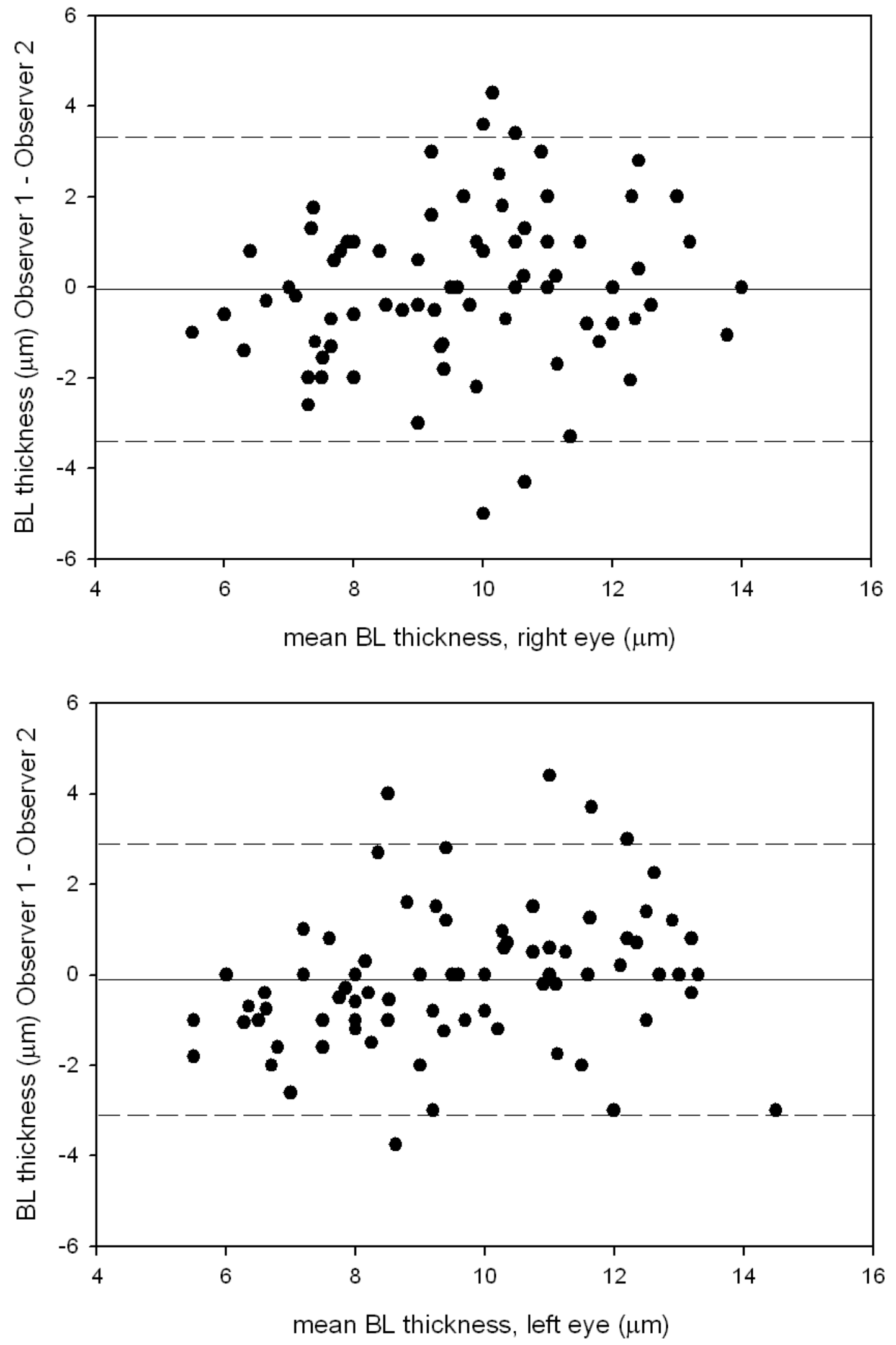

Figure 3. Bland-Altman plots of inter-observer agreement in BL thickness measurement. Each circle represents the eye of one subject. The solid horizontal line indicates the mean 
inter-observer difference while dashed lines indicate the lower and upper boundaries for the 95\% limits of agreement.
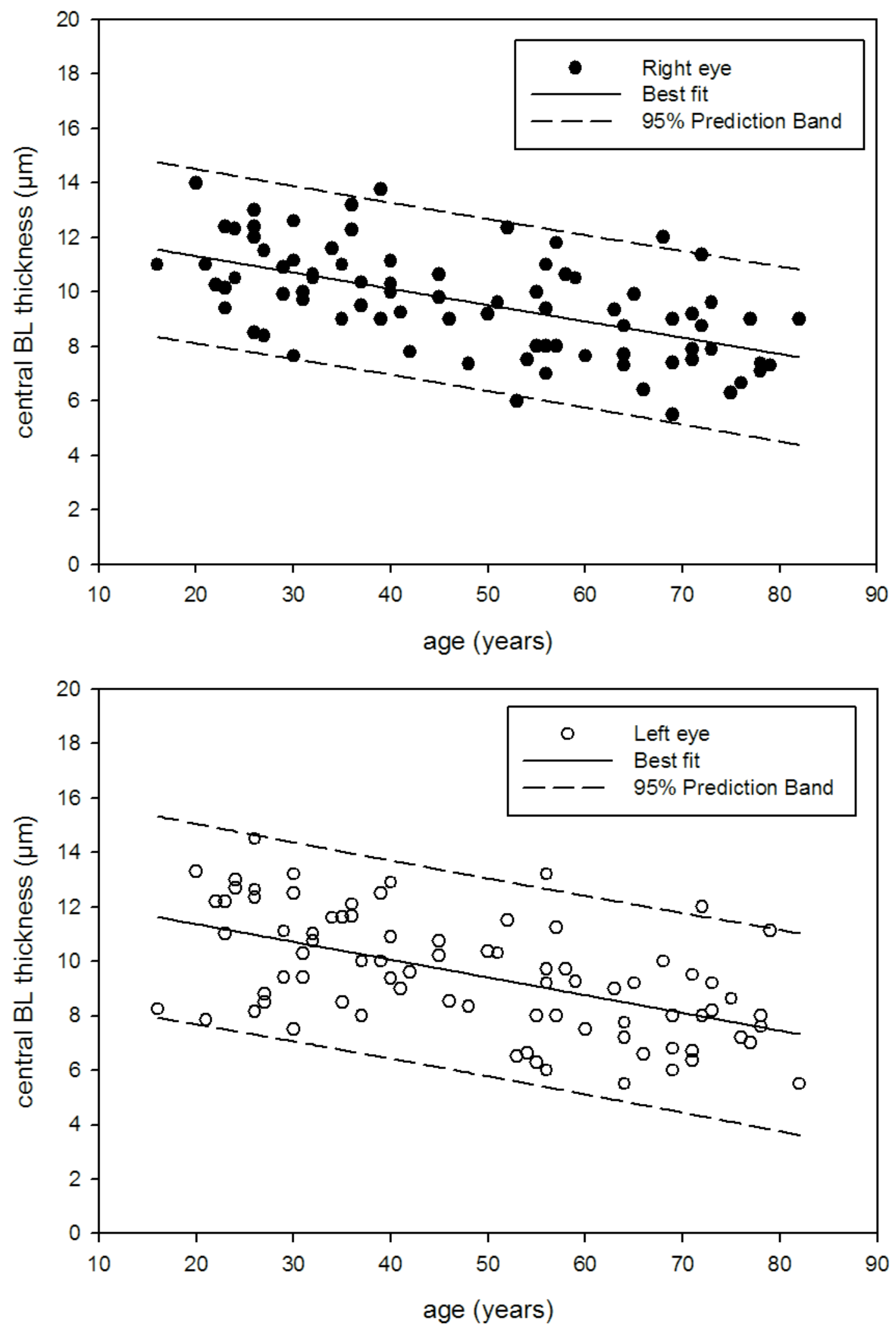
Figure 4. Decline in BL thickness with age in right (upper plot) and left (lower plot) eyes of healthy subjects. The solid line indicates the best-fit linear regression line, while dashed lines indicate the 95\% confidence interval for BL thickness. BL thickness declined significantly with increasing age (Pearson $\mathrm{P}<0.0001$ for right and left eyes). 